\title{
An investigation of low-mass-ratio EW systems from the Catalina Sky Survey
}

\author{
E. Lalounta ${ }^{1}$, A. Papageorgiou ${ }^{2,3}$, P.E. Christopoulou ${ }^{1}$ and \\ M. Catelan ${ }^{2,3}$ \\ 1 Department of Physics, University of Patras, Greece \\ 2 Pontificia Universidad Católica de Chile, Santiago, Chile \\ 3 Millennium Institute of Astrophysics, Santiago, Chile
}

Received: November 1, 2019; Accepted: February 20, 2020

\begin{abstract}
We have conducted a survey of overcontact binary systems (EW) with mass ratio $\leqq 0.25$ from the Catalina Sky Survey (CSS) that are considered strong merger candidates and are probable progenitors of FK Com-type stars and blue stragglers. The discovery of such extreme mass ratio overcontact binaries is vital to resolve the critical mass ratio ambiguity to merge, the mass loss process, and to refine the current theoretical models. So far only a few tens of such systems have been identified. To increase this sample, we selected and derived the physical parameters (mass, temperature and radius ratios, inclination and fill-out factor, along with their respective uncertainties) of 92 newly discovered totally eclipsing low-mass-ratio (LMR) EW systems based on their $V_{\text {CSS }}$ light curves, using PHOEBE-0.31a scripter and Monte Carlo methods.
\end{abstract}

Key words: binaries: close - binaries: evolution

\section{Introduction}

Eclipsing W UMa-type binaries (EW) are a unique class of eclipsing binaries. Their components are main-sequence stars of A-K spectral type and share a common convective envelope from where the energy transfer occurs. These systems are characterized by short periods in the range of 0.22 days to around 1.0 day. In addition, their components are of different mass (usually expressed by the mass ratio of the less massive over the more massive component, $q=\frac{M_{2}}{M_{1}}$ ). The minimum mass ratio $\left(q_{\min }\right)$ predicted by theoretical models is around $0.05-0.109$ (Li \& Zhang, 2006; Arbutina, 2009), depending on the adopted approximations. According to Kiseleva-Eggleton \& Eggleton (2001), EW systems with $q \leqq 0.25$ and fillout-factor ${ }^{1} \mathrm{FF} \geqq 50 \%$ are in the process of merging and they are probable progenitors of FK Com-type stars and blue stragglers.

${ }^{1} \mathrm{FF}=\frac{\Omega_{1}-\Omega}{\Omega_{1}-\Omega_{2}}$, where $\Omega$ is the value of the potential at the common surface of the binary and $\Omega_{1}, \Omega_{2}$ are the values of the potentials at the first and second Lagrangian point, respectively. 


\section{Identification of LMR EWs in the CSS}

In this study we used the light curves (LCs) of the 30,592 EW systems (sample 1) from the Catalina Sky Survey (CSS; Drake et al., 2014), combined with 44 LCs in the $V$ band of the confirmed LMR EW systems from the literature (sample 2). We first cleaned the light curves of sample 1 using a $3 \sigma$ clipping along the phase-folded LC by adopting a pre-defined period from Drake et al. (2014). The initial epoch values were determined using an iterative procedure of fitting a 2-degree polynomial to the deeper eclipse. We then applied a Fourier decomposition technique to the phase-folded, normalized flux LCs of both samples based on the following equation (Eqn. 4 in Deb \& Singh, 2009):

$$
m(t)=A_{0}+\sum_{j=1}^{10}\left\{a_{j} \sin [2 \pi j \phi(t)]+b_{j} \cos [2 \pi j \phi(t)]\right\} .
$$

Our investigation on the Fourier coefficient space showed that the higher-order Fourier coefficients, especially $b_{8}$, are more efficient in identifying LMR systems. As a result the initial sample 1 was reduced to 2,101 LMR candidates and finally to 92 , by visual inspection of the remaining LCs, focusing on totality and duration of the eclipses.

\section{Physical parameters}

To initialize the EB models, the systems' effective temperatures were adopted from the values given by Marsh et al. (2017) after cross-matching with our sample, resulting in 52 out of our 92 systems having a temperature determined in this manner. For the remainder of our sample, we averaged the temperatures from Gaia DR2 (Gaia Collaboration et al., 2018) and the $T_{\text {eff }}$ given by Pecaut \& Mamajek (2013) and corresponding to the $J-H$ color index (Cutri et al., 2003).

\subsection{Light Curve analysis using PHOEBE-scripter}

In order to estimate the mass ratios of the LMR candidates, a 2-dimensional grid was constructed in the mass ratio $(q)$ - inclination $(i)$ plane. The $q-i$ range was set to $[0.1-0.6]$ and $\left[68^{\circ}-90^{\circ}\right]$, with a step of 0.05 and $1^{\circ}$, respectively. Each pair of grid values was adopted and fixed to initialize each model. The $q-i$ scan method was performed using PHOEBE-scripter (Prša \& Zwitter, 2005) in "overcontact not in thermal contact" mode. Gravity darkening and limb darkening coefficients were adopted according to the systems' effective temperature, while for the latter the van Hamme (1993) tables were used. The adjusted parameters were the temperature of the secondary component $\left(T_{2}\right)$, the modified potential $\left(\Omega_{1,2}\right)$, and the passband luminosities. After the successful completion of the fitting for every grid pair, the ranges of the grid were constrained around 
the minimum $\chi^{2}$ value region and a second search began, with $q$-step value of 0.01 . The final model was constructed using the best $q-i$ pair and by adjusting also the inclination, the time of the primary eclipse, $\mathrm{HJD}_{0}$, and the period of the system.

As a test of our method, a synthetic light curve was generated using PHOEBEscripter that mimics the CSS photometric data $\left(V_{\text {err }} \sim 0.01 \mathrm{mag}\right.$ and $\sim 350$ data points) for a system of $q=0.096$ and $i=81^{\circ} 4$. The derived solution corresponded to a system of $q=0.1$ and $i=83^{\circ}$, which is in good agreement with the actual values.

As a result of the described method a final catalog with the physical parameters $\left(i, \frac{T_{2}}{T_{1}}, \frac{R_{2}}{R_{1}}, \mathrm{FF}, \mathrm{HJD}_{0}\right.$, period) of 92 new LMR EWs was created. A representative example of an LMR EB from CSS data given in Fig. 1

\subsection{Error estimation of the physical parameters}

The formal errors from the LC fitting procedure heavily underestimate parameter true uncertainties. Thus, the estimation of the uncertainty of the physical parameters was done by performing Monte Carlo simulations (Papageorgiou \& Christopoulou, 2015; Papageorgiou et al., 2019). A synthetic LC was created for each system from the observed one, by a random displacement of each point 1000 times, according to its photometric point error drawn from a normal distribution with zero mean and the photometric error as the standard deviation. Then, every synthetic LC was fitted by adjusting only $T_{2}, \Omega_{1,2}$, passband luminosity, and $i$. We finally extracted the lower and the upper error bounds from each parameter distribution $\left(\frac{T_{2}}{T_{1}}, \frac{R_{2}}{R_{1}}, \Omega_{1,2}, i\right)$. This is the final error that is provided for each of these quantities in our final catalog.
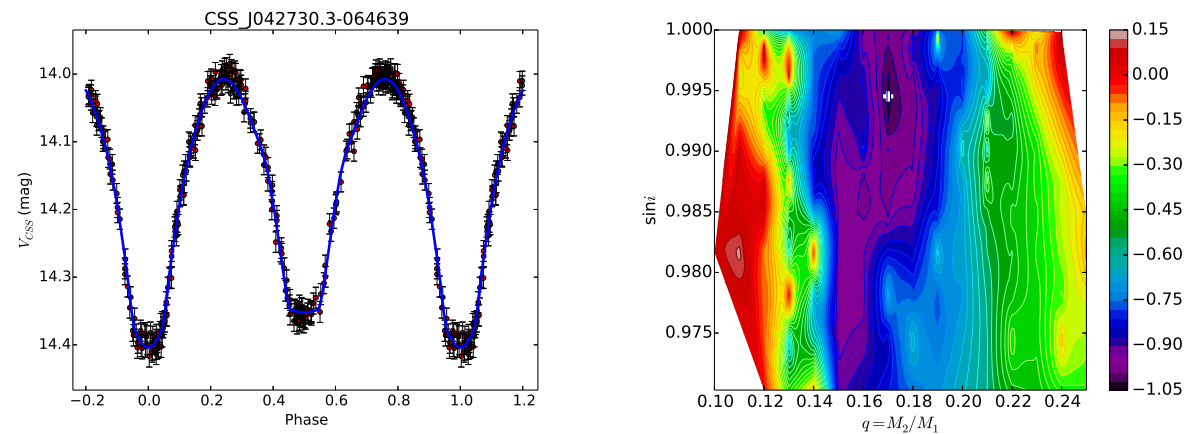

Figure 1. (left) Representative example of an EB from CSS data with $q=0.17$ and $i=83^{\circ}$ plotted with the best fitted model (blue line). (right) A contour plot of $\log \chi^{2}$ in the $(q, i)$ plane. The white cross denotes the final solution. 
Acknowledgements. A.P. and M.C. gratefully acknowledge the support provided by Fondecyt through grants \#3160782 and \#1171273. Additional support for this project is provided by the Ministry for the Economy, Development, and Tourism's Millennium Science Initiative through grant IC 120009, awarded to the Millennium Institute of Astrophysics (MAS); and by Proyecto Basal AFB-170002.

\section{References}

Arbutina, B., Possible solution to the problem of the extreme mass ratio W UMatype binaries. 2009, Mon. Not. R. Astron. Soc., 394, 501, DOI: 10.1111/j.13652966.2008.14332.x

Cutri, R. M., Skrutskie, M. F., van Dyk, S., et al. 2003, 2MASS All Sky Catalog of point sources.

Deb, S. \& Singh, H. P., Light curve analysis of variable stars using Fourier decomposition and principal component analysis. 2009, Astron. Astrophys., 507, 1729, DOI: 10.1051/0004-6361/200912851

Drake, A. J., Graham, M. J., Djorgovski, S. G., et al., VizieR Online Data Catalog: Catalina Surveys periodic variable stars (Drake+, 2014). 2014, VizieR Online Data Catalog, J/ApJS/213/9

Gaia Collaboration, Brown, A. G. A., Vallenari, A., et al., Gaia Data Release 2. Summary of the contents and survey properties. 2018, Astron. Astrophys., 616, A1, DOI: 10.1051/0004-6361/201833051

Kiseleva-Eggleton, L. \& Eggleton, P. P., Binary Orbits in Triple Systems. 2001, in Astronomical Society of the Pacific Conference Series, Vol. 228, Dynamics of Star Clusters and the Milky Way, ed. S. Deiters, B. Fuchs, A. Just, R. Spurzem, \& R. Wielen, 488

Li, L. \& Zhang, F., The dynamical stability of W Ursae Majoris-type systems. 2006, Mon. Not. R. Astron. Soc., 369, 2001, DOI: 10.1111/j.1365-2966.2006.10462.x

Marsh, F. M., Prince, T. A., Mahabal, A. A., et al., Characterization of 9380 contact binaries from the CRTS Variable Sources Catalogue. 2017, Mon. Not. R. Astron. Soc., 465, 4678, DOI: 10.1093/mnras/stw2110

Papageorgiou, A., Catelan, M., Christopoulou, P.-E., Drake, A. J., \& Djorgovski, S. G., Physical Parameters of Northern Eclipsing Binaries in the Catalina Sky Survey. 2019, Astrophys. J., Suppl., 242, 6, DOI: 10.3847/1538-4365/ab13b8

Papageorgiou, A. \& Christopoulou, P. E., Absolute Parameters and Physical Nature of the Low-amplitude Contact Binary HI Draconis. 2015, Astron. J., 149, 168, DOI: 10.1088/0004-6256/149/5/168

Pecaut, M. J. \& Mamajek, E. E., Intrinsic Colors, Temperatures, and Bolometric Corrections of Pre-main-sequence Stars. 2013, Astrophys. J., Suppl., 208, 9, DOI: 10.1088/0067-0049/208/1/9

Prša, A. \& Zwitter, T., A Computational Guide to Physics of Eclipsing Binaries. I. Demonstrations and Perspectives. 2005, Astrophys. J., 628, 426, DOI: $10.1086 / 430591$ 
van Hamme, W., New Limb-Darkening Coefficients for Modeling Binary Star Light Curves. 1993, Astron. J., 106, 2096, DOI: 10.1086/116788 